\title{
SSLDoc: Automatically Diagnosing Incorrect SSL API Usages in C Programs
}

\author{
Zuxing Gu, Jiecheng Wu, Chi Li, Min Zhou, Ming Gu \\ School of Software Engineering, Tsinghua University, Beijing , China, 100084
}

\begin{abstract}
Secure Sockets Layer (SSL) and Transport Layer Security (TLS) protocols provide a reliable communication channel between applications over the Internet. Implementations of these protocols (e.g., OpenSSL and GnuTLS) publish wellformat documentation and examples online to guide the usage of SSL/TLS APIs. However, incorrect usages have caused many severe vulnerabilities (e.g., privilege escalation, denial of service, man-in-the-middle attack, etc.) in recent years. In this paper, we introduce SSLDoc to diagnose incorrect SSL API usages in real-world $C$ programs automatically. The key insight behind SSLDoc is a constraint-directed static analysis technique powered by domain-specific usage patterns that we learn from real-world vulnerabilities and bug-fix-related patches. We have instantiated SSLDoc for OpenSSL APIs and applied it to large-scale opensource programs. SSLDoc found $\mathbf{4 5}$ previously unknown securitysensitive bugs in OpenSSL implementation and applications in Ubuntu. We created and submitted issues for all of them. Up to now, 35 have been confirmed by the corresponding development communities and 27 have been fixed in master branch.

Index Terms-SSL, API usage validation, static analysis, bug detection
\end{abstract}

\section{INTRODUCTION}

Secure Socket Layer (SSL) and Transport Layer Security (TLS) are the most widely deployed protocols in securitysensitive software. They provide a confidential and authentic end-to-end communication mechanism against an active, manin-the-middle attacker. The details of these protocols are complicated and involves many steps to set up and validate certificate authority [1], [2]. Therefore, client programs usually rely on SSL libraries such as OpenSSL [3] and GnuTLS [4], which encapsulate the internal details and diverse kinds of cryptography algorithms into APIs with well-format documentation and examples. However, correct usage of SSL APIs is required to satisfy certain constraints, such as call conditions or call orders. Violations of these constraints will lead to software bugs and more critically, can have severe security implications. For example, missing error status code validation of SSL APIs will cause a denial of service by remote attackers (CVE-2016-2182 [5]), and broken SSL certificate validation will result in man-in-the-middle attacks [6]. A recent study show that SSL certificate validation is completely broken in many security-critical applications and libraries [7].

Many different tools, techniques and methodologies have been proposed to address the above problems. Clark et al. [8] present a comprehensive survey of SSL issues to enhance the certificate infrastructure used in practice. Brubaker et al. [9]

DOI reference number: 10.18293/SEKE2019-006 systematically test the correctness of the certificate validation logic in SSL/TLS implementations. However, they focus on SSL implementation and require considerable manual efforts to prepare a test environment.

To automatically detect incorrect usages of SSL APIs in client programs, static analysis has long prevailed as one of the most promising techniques [10]. For example, He et al. [11] design and implement SSLINT, a scalable static analysis tool to match a program dependence graph with a handcrafted, precise signature modeling the correct logic usage of SSL APIs. Although SSLINT is capable of detecting incorrect usages in practice, it is hard to apply to APIs without predefined signatures and produces many false positives and false negatives due to imprecise static analysis (e.g., flow-insensitive and context-insensitive). Yun et al. [12] present APISan for incorrect API usages of causal relation and semantic relation on arguments with security implications by leveraging the strength of static analysis (such as control dependency analysis) and code mining (such as frequent sub-itemsets mining algorithm). It provides accurate detection and can be applied to scale real-world system programs. However, a challenge for such tools is insufficient data to train models, which is particularly severe for SSL APIs in client programs.

In this paper, we aim at augmenting current detection capability of incorrect SSL API usage for large-scale C programs. The key insight is a constraint-directed static analysis technique powered by domain-specific usage patterns. To understand the root causes of incorrect usages of SSL APIs, we begin with a preliminary investigation of real-world vulnerabilities to summarize generic incorrect usage patterns. Leveraging this knowledge, we design and implement SSLDoc, a static analysis detector employing under-constrained symbolic execution [13] to generate abstract symbolic traces with rich semantics and detect incorrect usages. In this way, SSLDoc can precisely conduct a flow-, control- and context-sensitive analysis inter-procedurally (i.e., capable of capturing temporal sequencing of API calls, path constraints, and data flows between parameters and return values in or across procedures).

To evaluate SSLDoc in practice, we instantiated it with OpenSSL APIs and applied it to more than half million lines of source code, including OpenSSL implementation and 15 applications in Ubuntu. The result shows that SSLDoc discovers 45 previously unknown security-sensitive incorrect SSL API usages. We reported our findings to developers and received 35 confirmations, out of which 27 have been fixed in multiple branches. Moreover, we share the lessons 
learned from bug detecting, issue reporting and discussions with developers.

In summary, our paper makes the following contributions:

- We design and implement SSLDoc, a static analysis tool to augment current detection capability of incorrect SSL API usage for large-scale $\mathrm{C}$ programs.

- We instantiate SSLDoc with OpenSSL APIs and apply it to real-world programs. It discovers 45 previously unknown incorrect SSL API usages, out of which 35 have been confirmed by developers.

- We share the lessons learned from bug detecting, issue reporting and discussions with developers in practice. We hope our findings can motivate more researcher to combat incorrect SSL API usages.

The rest of this paper is organized as follows. Section II provides motivating examples of our work. Section III presents the design of SSLDoc, followed by an evaluation in Section IV. We share the lessons learned in Section $\mathrm{V}$ and discuss related work in Section VI and conclude in Section VII.

\section{Motivating EXAMPLE}

Instead of implementing SSL themselves, Client programs usually rely on APIs of SSL libraries such as OpenSSL and GnuTLS as well as higher-level data-transport libraries such as Curl [14]. While APIs encapsulate the details, they also expose rich semantic constraints. Violations of these constraints, in turn, lead to serious security problems.

To better understand incorrect SSL API usage patterns and how developers fix them in practice, we manually studied four years' (from 2013 to 2017) CVE entries related to API usage bugs in National Vulnerability Database ${ }^{1}$. They are extracted through approximate keywords matching (e.g., "OpenSSL API usage" and "incorrect SSL usage") and contain concrete patches to fix the bugs. We investigate both the CVE description messages and patches, and identify two generic incorrect usage patterns as shown in Figure 1:

- Certificate Validation. SSL libraries encapsulate the core functionality of protocols and export APIs to utilize the implementation. However, the client needs to validate all kinds of certificates in applications. Missing validations might allow attackers to cause a denial of service or man-inthe-middle via an invalid one. Figure 1a shows an example of such vulnerabilities reported in CVE-2015-0288 [15]. Function X509_get_pubkey ( ) ${ }^{2}$ attempts to decode the public key for $x$. If an error occurs, it will return NULL. In function $\times 509$ to $\times 509 \mathrm{REQ}()$, the return value pktmp is used without checking the error code, which results in a NULL Pointer Dereference bug. Beyond null pointer checking, SSL libraries use various error protocols in practice (e.g., 0 or negative for errors in OpenSSL, but -1 to -403 in GnuTLS).

- Causal Function Calling SSL libraries allocate memory resources for cryptography algorithm computing, which

\footnotetext{
${ }^{1}$ http://cve.mitre.org/

${ }^{2} \mathrm{https} / / /$ www.openssl.org/docs/manmaster/man3/X509_get_pubkey.html
}

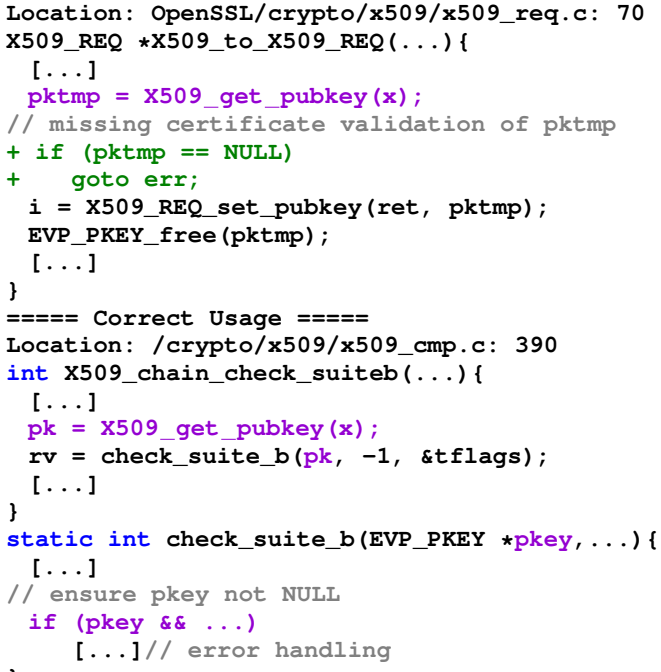

(a) Incorrect usage for missing certificate validation reported in CVE-2015-0288 [15].

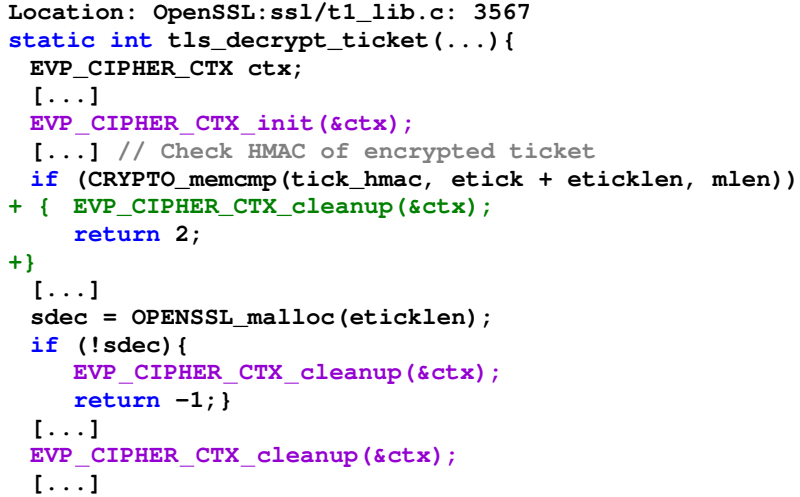

(b) Incorrect usage for missing releasing resource reported in CVE2014-3567 [16].

Fig. 1: Motivating examples of incorrect SSL API usages.

should release after their lifecycle by invoking a causal function calling. Violations of such causal relation (i.e., $a-b$ pattern) will cause a denial of service (memory consumption) via an intentionally crafted input by remote attackers. For example, EVP_CIPHER_CTX_cleanup ( $)^{3}$ clears all information from a cipher context ctx and free up any allocated memory associated with it. However, missing invoking it along the error handling path of tls_decrypt_ticket ( ) will be exploited by a crafted session ticket that triggers an integrity-check failure as shown in Figure 1b.

Detection of the above bugs is not trivial. It requires a wide spectrum of semantics instead of simply syntactic matching. For example, function invocation of X509_get_pubkey () at Line 16 in Figure 1a is correct, because check_suite_b () validates the first parameter to ensure that pkey is not NULL. To filter out such instance, it

\footnotetext{
${ }^{3}$ https://www.openssl.org/docs/man1.0.2/crypto/EVP_CIPHER_CTX cleanup.html
} 


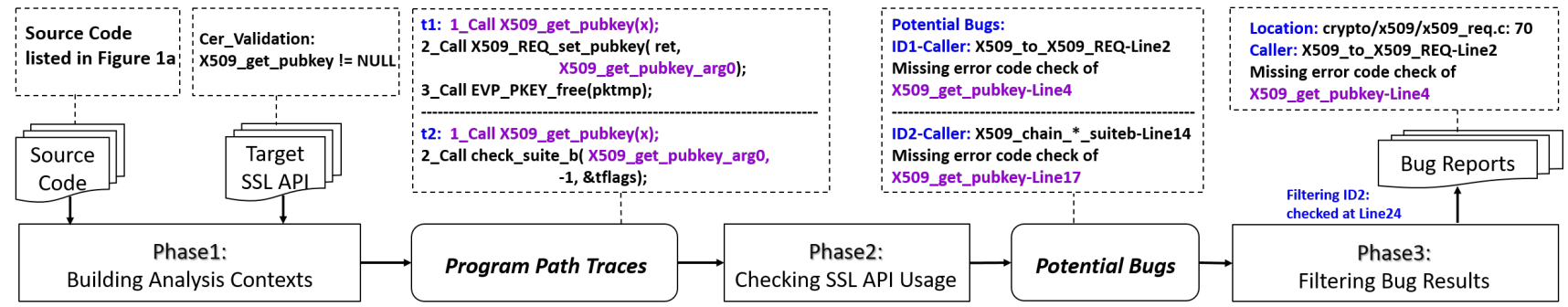

Fig. 2: Overview of SSLDoc's workflow.

demands a flow- and context-sensitive semantic analysis interprocedurally. Moreover, path-sensitive analysis also should be taken into consideration to check memory leak along different error handling paths (e.g., Line 14 and 17 in Figure 1b).

\section{APPROACH}

In this section, we introduce SSLDoc, a static analysis tool to augment current SSL API usage detection capability for large-scale $\mathrm{C}$ programs. We first present a brief overview of our approach with an example of Figure 1a and elaborate each step of bug detection in the following parts.

As shown in Figure 2, SSLDoc takes the source code and target APIs as input and generates bug reports with concrete locations and reasons as output. Bug detection consists of three basic steps. (1) In Phase-1, the analysis context is built by constructing the control flow graph and creating program path traces for each target API by employing under-constrained symbolic execution. In this example, two traces, $\mathrm{t} 1$ and $\mathrm{t} 2$, are generated, as shown in the box above Program Path Traces. In this way, SSLDoc can successfully capture the usage context of X509_get_pubkey (), EVP_PKEY_free () and those in between. (2) In Phase-2, SSLDoc employs the traces to detect violations of API usages as potential bugs. For example, two API-misuse instances of X509_get_pubkey () are found for missing certificate validations labeled as $\mathrm{Po}$ tential Bugs. (3) In Phase-3, SSLDoc improves the detection precision by leveraging inter-procedural semantics and usage statistics. Then, the second misuse is filtered out for the check conducted in the X509_to_X509_REQ() at Line-24. We discuss the details of our approach as follow.

\section{A. Building Analysis Contexts}

SSLDoc performs symbolic execution to generate program path traces that capture rich semantic information for each

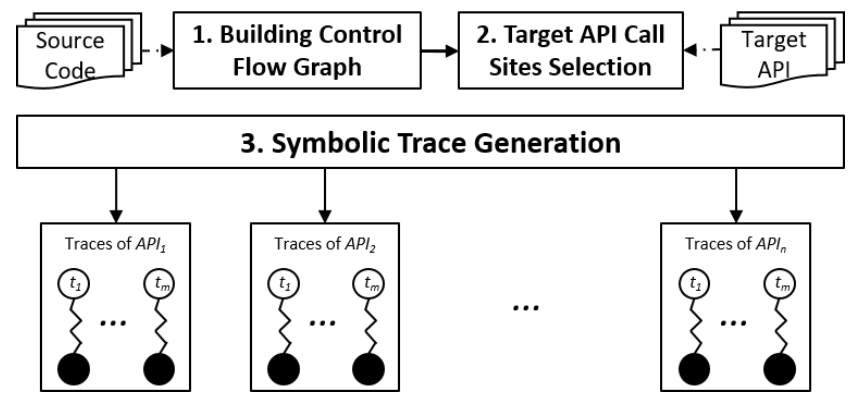

Fig. 3: Workflow of building analysis contexts. target API. In Figure 3, we illustrate the workflow for building analysis context, which consists of three steps. First, SSLDoc parses the source code and builds a control flow graph (CFG). Then, for each target API $f$, we select analysis entries as target API call sites by labeling the callers $C$, which invokes $f$. Next, for each caller $c \in C$, symbolic execution is employed to generate a series of program path traces $T$ with rich semantics of usages of $f$ while traversing the CFG.

We use $\mathbb{N}, \mathbb{Z}$ to denote the set of non-negative and all integers, respectively. In Figure 4, we formally describe the structure of program path traces computed by SSLDoc, where $i d \in \mathbb{N}, n \in \mathbb{N}, z \in \mathbb{Z}$ and $a p$ is short for Accesspath [17] to represent memory locations in the form of regular expressions. Each trace $t$ consists of a sequence of actions $a^{+}$with a value map $V$. In particular, Assume action is used to capture path-sensitive semantics. All the actions are labeled while traversing CFG to support flow-sensitive analysis. $V$ records the semantics from a symbolic variable $s v$ to a concrete value $c v$. A symbolic variable is defined by an action labeled by $i d$ and the index $n$. For example, $i d \_f \_$arg_ $i$ denotes the $i^{t h}$ parameter of $f$ called in the $i d^{t h}$ action. In this way, we can capture the invocation context semantics. We use $f \_$arg_0 to represent the return value of $f$ and arg_ 0 for the symbolic variable returned by the caller $c$ of $f$ in Return action. Therefore, our program path trace is capable of capturing the flow-, context- and path-sensitive semantics.

In Figure 5, We illustrate three traces of the example code listed in Figure 1a. $t_{1}$ and $t_{3}$ are original code snippets, and $t_{2}$ is with the bug-fix patch. All traces start from the action calling $X_{-} 509$ _get_pubkey (). Then, $t_{1}$ directly passes the return value 1_X509_get_pubkey_arg_0 into X509_REQ_set_pubkey () without certificate validation. By contrast, $t_{2}$ validates the return value immediately. Even though $t_{3}$ passes it into check_suite_b() without valida-

$$
\begin{aligned}
\text { (Traces) } T: & :=t \\
\text { (trace) } t & :=\left(i d \_a\right)^{+} ; V \\
\text { (action) } a & :=\operatorname{Assume}(\exp ) \mid \text { Call } f\left(s v^{*}\right) \mid \operatorname{Return}(s v) \\
\text { (expression) exp }: & :=s v 1 \text { cmpop sv2 } \\
\text { (value map) } V: & :=s v \rightarrow c v \\
\text { (symbolic variable) } s v & :=\left(i d \_f \_ \text {arg } n\right) \\
\text { (compare operator) cmpop }: & :=!=|==|>=|>|<=\mid< \\
\text { (concrete value) } c v & :=z \mid \text { ap } \mid \text { NULL } \\
\text { (function) } f & \in \mathbb{F}
\end{aligned}
$$

Fig. 4: Abstract syntax of program path traces. 


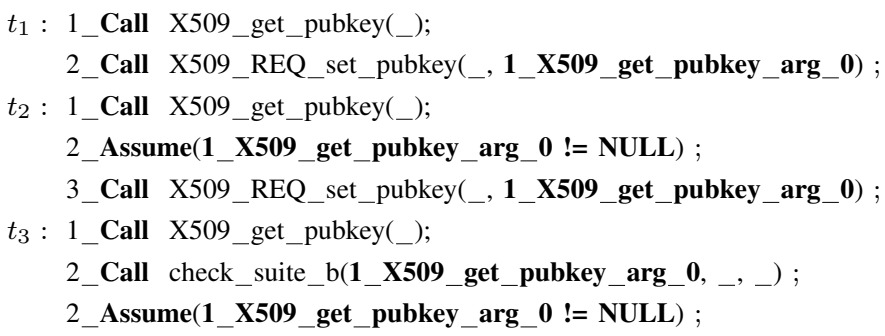

Fig. 5: Program path traces of the code in Figure 1a, where we use "_" to represent values irrelevant.

tion, check_suite_b () checks the first parameter at Line 24 in Figure 1a.

Similar to the traditional analysis, the key challenge of building such path traces in large and complex programs is to overcome the path-explosion problem. We make two design decisions to achieve scalability without sacrificing substantial accuracy. (1) Limiting inter-procedural analysis. SSLDoc performs symbolic execution intre-procedurally for each caller $c$ of the target API $f$ at most two depth (i.e. we track $c$ and callees of $c$ ). We refine the bug detection results by a filtering phase with the deeper inter-procedural semantics presented in Section III-C. (2) Unrolling loops. SSLDoc unrolls each loop only once to reduce the number of paths explored. While this restriction can limit the accuracy of the semantic computation, it does not noticeably affect the accuracy of SSL API bug detection for only a small number of usages related to loop variables.

\section{B. Checking API misuses}

In the checking phase, SSLDoc employs target APIs and the program path traces $T$ to detect bugs. To configure usage pattern of SSL APIs, we provide each target $f$ with a usage pattern type $f . \mathcal{T}$, which can be certificate validation with a predicate $\mathcal{P}$ (e.g., X509_get_pubkey $!=$ NULL) and causal function calling with a usage pattern $\mathcal{C}$ (e.g., EVP_CIPHER_CTX_init $(\arg 1) \rightarrow$ EVP_CIPHER_CTX_cleanup(arg1), where $\arg 1$ labels the target memory object). We illustrate our detecting algorithm in Algorithm 1. First, we extract all the target functions into APISet. For each API $f$, we detect API-misuse bugs along the traces $T^{\prime}$ that invoke $f$. Then, for each trace $t$ in $T^{\prime}$, we validate whether the usage pattern in $f . \mathcal{T}$ are satisfied along $t$. If a path fails, SSLDoc labels the call site of $f$ along this $t$ as a potential bug. To check the satisfaction of certificate validation along $t$, we compute the satisfiability of $\mathcal{P}$. That is, whether there is an Assume action to ensure $\mathcal{P}$. For usage pattern $\mathcal{C}$, we match with Call actions, which satisfy the target memory object constraint. If any of the constraints fail to match, SSLDoc reports a bug. For example, $t_{1}$ in Figure 5 fails to ensure the certificate validation X509_get_pubkey != NULL, which may result in a null pointer dereference bug.

\section{Filtering Bug Reports}

To achieve the scalability required to support real-world programs, we employ a limiting inter-procedural strategy to ad-

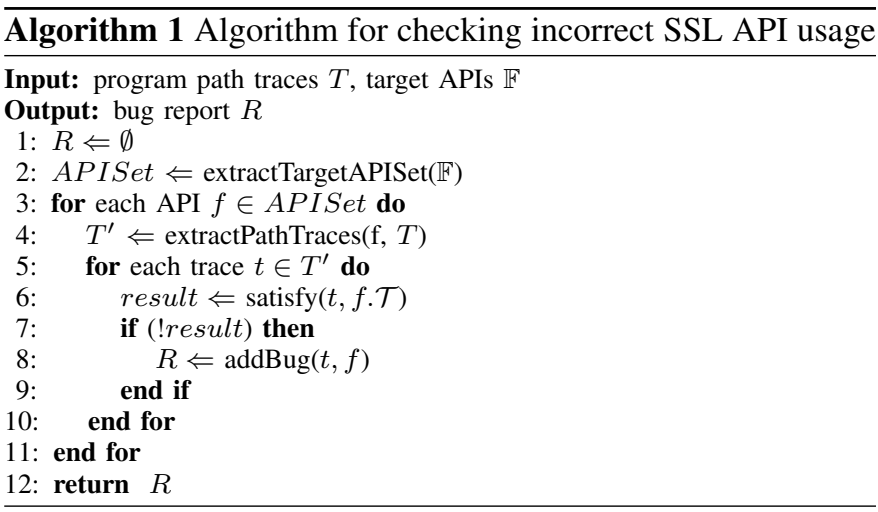

dress the path-explosion problem. The strategy generates false positives when a usage cross more than two functions. However, developers dislike using tools with low precision [18]. Therefore, we apply deeper inter-procedural semantics and rank the final results according to usage statistics.

First, we conduct semantic-based filtering. We attempt to infer semantics acrossfunctions. For the missing validation of certificate $x$, we further check the functions which directly receive $x$ as a parameter. If these functions contain sanity check against $x$, we filter it out. For causal function calling pattern as $a \rightarrow b$, if the target memory object of $a$ is directly assigned to the parameter of the caller $c$ of $a$ or returned by $c$, we check whether callers $\mathbb{C}$ of $\mathrm{c}$ invoke $b$. We filter out the cases that contain function invocation of $b$.

Then, we conduct a usage-based ranking. Basically, we compute the number of correct/incorrect usage traces respectively, ranks bug reports in decreasing order of their likelihood of being bugs as $\mathcal{H}(f)=\frac{\text { \#of correct usage traces of } f}{\text { \#of incorrect uage traces of } f}$. The highest likelihood value indicates that more correct usages occur and the violations are less. Therefore, the violations are highly buggy. Note that, we use the trace number instead of call site number, because of the observation that many bugs occur along path-branches with different context semantics. However, we have to specially treat when $\mathcal{H}(f)$ is 0 , because it indicates that all the usages are buggy. The preliminary experiment results show that it frequently occurs in small programs which invoke SSL APIs only once or twice.

\section{Implementation}

SSLDoc is built in Java language. We preprocess the source code into LLVM-IR $3.9^{4}$, which provides a typed, static single assignment (SSA) and well-suited low-level language. Then, we parse the LLVM-IR by javacpp ${ }^{5}$ and construct an extended control flow graph, which classifies the edges into control edges for semantic computation and summary edges to provide a mechanism to support large-scale programs. We have integrated part of OpenSSL APIs with SSLDoc and provide an interface to extend our analysis in a human-readable format named Yaml ${ }^{6}$.

\footnotetext{
${ }^{4}$ http://releases.llvm.org/3.9.0/docs/ReleaseNotes.html

${ }^{5}$ https://github.com/bytedeco/javacpp

${ }^{6}$ http://yaml.org/
} 


\section{Evaluation}

In this section, we describe our results from incorrect SSL API usage detection on large-scale open-source programs using SSLDoc. We begin by providing the experimental setup. Then we present the security-sensitive bugs we found and concluding with lessons we learned.

a) Experimental Setup: We applied SSLDoc to find incorrect SSL API usages in OpenSSL implementation as well as applications using OpenSSL library in Ubuntu 16.04. Target applications are selected by search dependence attributes using package management command line "apt-cache rdepends libssl1.0.07". In total, we found more than 1200 packages using this library and selected 15 packages which are open-source on Github and ongoing development. For all the 16 programs, we detect incorrect usages in the latest stable versions. Then, we use GNU cflow $^{8}$ to extract target SSL APIs invoked in the applications and create usage pattern mentioned in Section II according to the user manual of OpenSSL ${ }^{9}$. In total, 136 different SSL APIs are integrated with SSLDoc. We ran SSLDoc on Ubuntu 16.04 LTS (64-bit) with a Core i5-4590@3.30 GHz Intel processor and 16 GB memory.

b) Result: Overall, SSLDoc detected 45 previously unknown security-sensitive incorrect SSL API usages as listed in Table I. We tried our best to understand the context and created issues for all the bugs to the developers of each program. Up to now, 32 of the new bugs have been confirmed by the developers and 27 have been fixed in the master branch.

For example, in Figure 6 we present a bug caused by incorrect validation of connect status in dma, a small Mail Transport Agent, which is fixed at 12 hours after we submitted the bug report with bug description and explanation of bug traces. Function SSL_connect ( ) initiates the SSL handshake with a server. It returns 0 and negative integers to indicate SSL handshake is not successful. However, the status validation in dam/crypto.c only checked against negative integers, which may cause a man-in-the-middle attack leading to leakage of user credentials and emails messages.

\section{Discussion}

While investigating the bug reports generated by SSLDoc, we find several intricate bugs and gain useful experience in the bug reporting process with open-source developers. We share our following experience. (1) Incorrect SSL API usages are not corner cases. In total, we find 45 previously unknown incorrect usages. However, OpenSSL library has provided well-format documentation and examples to guide correct usages. These bugs may result from the lack of a bug information sharing mechanism and the lack of API usage constraints among client software developers. We believe that bug fixing is an essential activity during the entire life cycle of software development. Automatic bug-finding tools,

\footnotetext{
${ }^{7}$ In Ubuntu16.04 OpenSSL library is listed as libss11.0.0.

${ }^{8}$ http://www.gnu.org/software/cflow/

${ }^{9}$ https://www.openssl.org/docs/manmaster/man3/
}

TABLE I: Previously unknown incorrect SSL API usages detected by SSLDoc

\begin{tabular}{|c|c|c|c|c|}
\hline Index & Program & Issue ID & Target API & Status \\
\hline 1 & \multirow{17}{*}{$\begin{array}{l}\text { OpenSSL } \\
\text { 1.1.1-pre8 }\end{array}$} & 6567 & RAND bytes & $\checkmark \checkmark$ \\
\hline 2 & & 6568 & ASN1 INTEGER get & $\checkmark$ \\
\hline 3 & & 6569 & ASN1 INTEGER set & $\checkmark \checkmark$ \\
\hline 4 & & 6570 & ASN1_object_size & $\checkmark$ \\
\hline 5 & & 6572 & BN_set_word & $\checkmark \checkmark$ \\
\hline 6 & & 6573 & HMAC_Init_ex & $\checkmark$ \\
\hline 7 & & 6574 & EVP_PKEY_get0_DH & $\checkmark \checkmark$ \\
\hline 8 & & 6575 & EC_KEY_generate_key & $\checkmark$ \\
\hline 9 & & 6781 & EC GROÜP new by curve name & $\checkmark \checkmark$ \\
\hline 10 & & 6789 & \begin{tabular}{|l|l} 
ASN"1_INTEGER_set \\
\end{tabular} & $\checkmark \checkmark$ \\
\hline 11 & & 6820 & ASN1 INTEGER to BN & $\checkmark \checkmark$ \\
\hline 12 & & 6822 & BN_sub & $\checkmark \checkmark$ \\
\hline 13 & & 6973 & EVP_MD_CTX_new & $\checkmark \checkmark$ \\
\hline 14 & & 6977 & ASN1_INTEGER_set & $\checkmark \checkmark$ \\
\hline 15 & & 6982 & OBJ_n̄id2obj & $\checkmark \checkmark$ \\
\hline 16 & & 6983 & BN_sub & $\checkmark \checkmark$ \\
\hline 17 & & 7235 & DH set0 key & $\checkmark$ \\
\hline 18 & dma & 59 & SSL connect & $\checkmark \checkmark$ \\
\hline 19 & \multirow{2}{*}{ exim } & 2316 & X509_NAME_oneline & $\checkmark \checkmark$ \\
\hline 20 & & 2317 & SSL_CTX_set_cipher_list & $\checkmark \checkmark$ \\
\hline 21 & \multirow{2}{*}{ hexchat } & 2244 & BN_set_word & $\checkmark$ \\
\hline 22 & & 2245 & DH_set0_key & $P$ \\
\hline 23 & httping & 41 & SSL_CTX_new & $\checkmark$ \\
\hline 24 & ipmitool & 37 & MD2 Init & $\checkmark$ \\
\hline 25 & \multirow{2}{*}{ open-vm-tools } & 291 & SSL_CTX_set_cipher_list & $\checkmark \checkmark$ \\
\hline 26 & & 292 & X509_STORE_CTX_get_current_cert & $\checkmark \checkmark$ \\
\hline 27 & \multirow{2}{*}{ irssi } & 943 & SSL_get_peer_certificate & $P$ \\
\hline 28 & & 944 & BIO_read & $P$ \\
\hline 29 & \multirow{2}{*}{ keepalive } & 1003 & SSL_CTX_new & $\checkmark \checkmark$ \\
\hline 30 & & 1004 & SSL_new & $\checkmark \checkmark$ \\
\hline 31 & \multirow{2}{*}{ thc-ipv6 } & 28 & BN_new & $\checkmark \checkmark$ \\
\hline 32 & & 29 & BN_set_word & $\sqrt{ }$ \\
\hline 33 & \multirow{2}{*}{ FreeRADIUS } & 2309 & BIO_new & $\checkmark \checkmark$ \\
\hline 34 & & 2310 & i2a_ĀASN1_OBJECT & $\checkmark \checkmark$ \\
\hline 35 & \multirow{3}{*}{ trafficserver } & 4292 & SSL_CTX_new & $P$ \\
\hline 36 & & 4293 & SSL_new & $P$ \\
\hline 37 & & 4294 & SSL_write & $P$ \\
\hline 38 & \multirow{2}{*}{ tinc } & 205 & BN_hex $2 b n$ & $\checkmark \checkmark$ \\
\hline 39 & & 306 & RAN̄D_load_file & $\checkmark \checkmark$ \\
\hline 40 & \multirow{2}{*}{ sslsplit } & 224 & SSL_CTX_use_certificate & $\checkmark \checkmark$ \\
\hline 41 & & 225 & SSL_CTX_use_PrivateKey & $\checkmark \checkmark$ \\
\hline 42 & \multirow{2}{*}{ rdesktop } & 280 & BN_bin2bn & $P$ \\
\hline 43 & & 281 & BN_mod_exp & $P$ \\
\hline 44 & \multirow{2}{*}{ proxytunnel } & 36 & SSL_connect & $P$ \\
\hline 45 & & 37 & SSL_new & $P$ \\
\hline
\end{tabular}

$\checkmark \checkmark$ is fixed, $\checkmark$ is confirmed without a patch, and $P$ is wating developr responce.

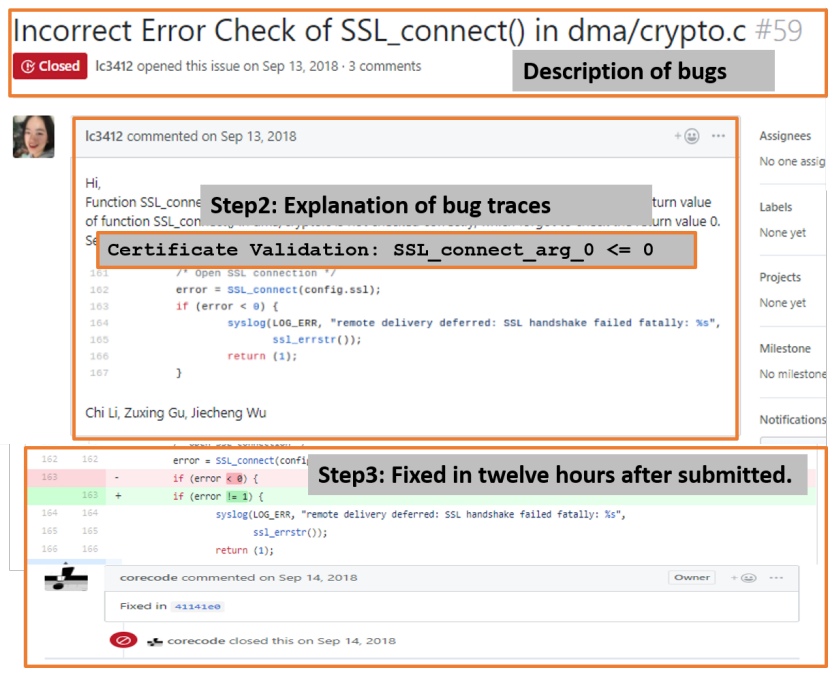

Fig. 6: Screenshot of a bug caused by incorrect validation of SSL_connet() status in dma, which is fixed at 12 hours. 
such as SSLDoc, with large-scale analysis capability can be integrated into the development cycle. In addition, SSLDoc can be customized to incrementally address this problem. (2) Accelerating manual auditing. SSL API usages usually have similar behavior patterns. For example, many types of vulnerabilities result from insufficient validation of input or missing certificate validations. However, discovering all the missing checks by human is tedious and time-consuming. Automatic tools can efficiently accelerate the manual auditing with differences extracted as good usages and bad usages. For example, two of the API misuses were fixed within 12 hours after we created the issues with possible fixing patches, as shown in Figure 6. (3) Intentional choices. We also find that many incorrect usages are not mistakes but intentional choices. Many error status code checks of return values are ignored by developers. During the bug reporting process with the OpenSSL developers, we learned that they intentionally ignore some error code checks for performance considerations or due to the lack of an error handling mechanism in $\mathrm{C}^{10}$.

\section{RELATED WORK}

A few works in the past have analyzed application vulnerabilities due to improper usage of SSL/TLS. Georgiev et al. [7] employ dynamic analysis to conduct MITM attacks and demonstrate that SSL certificate validation is completely broken due to badly designed APIs of SSL implementations. Later, Clark et al. [8] present a comprehensive survey of SSL security and Brubaker et al. [9] apply Frankencerts, a smart fuzzer to test SSL/TLS certificate validation code in implementation. He et al. [11] develop SSLINT, a scalable, automated, static analysis system for detecting incorrect certificate validation vulnerabilities in client programs with predefined API signatures. To automatically infer usage pattern, Yun et al. [12] present APISan to infer correct API usages from source code without manual effort and detect various properties with security implications. Moreover, generic bug detection approaches also can be applied to SSL/TLS API usage, such as static analysis approaches [19], [20] and testing [21]. SSLDoc specifically targets SSL API usages in C programs and complements these works. In addition, our work can be easily extended to other domains.

\section{CONCLUSION}

Client programs rely on APIs of libraries implementing SSL/TLS protocols to ensure reliable communications. Incorrect usage of such APIs will cause security-sensitive problems, even severe vulnerabilities. In this paper, we present SSLDoc, a static analysis detector to automatically diagnose incorrect usages of SSL APIs in C programs. We instantiate SSLDoc with APIs of OpenSSL and apply it to large-scale programs. We find 45 previously unknown bugs in OpenSSL implementation and 15 applications in Ubuntu which use SSL APIs, out of which 27 have been fixed. We share the lessons learned from bug detection and discussions with developers to

\footnotetext{
${ }^{10}$ https://github.com/openssl/openssl/issues/6575
}

motivate more researchers and practicers to combat incorrect SSL API usages.

\section{ACKNOWLEDGMENT}

We thank the anonymous reviewers for their helpful feedback. This research is sponsored in part by National Natural Science Foundation of China (Grant No. 61802259, 61402248, 61527812 ), National Science and Technology Major Project of China (Gran No. 2016ZX01038101), and the National Key Research and Development Program of China (Grant No. 2015BAG14B01-02, 2016QY07X1402).

\section{REFERENCES}

[1] T. Dierks and E. Rescorla, "The transport layer security (tls) protocol version 1.2," Tech. Rep., 2008.

[2] A. Freier, P. Karlton, and P. Kocher, "The secure sockets layer (ssl) protocol version 3.0," Tech. Rep., 2011.

[3] "Openssl: cryptography and ssl/tls toolkit." https://github.com/openssl/ openssl, 2019.

[4] "Gnutls: a secure communications library implementing the ssl, tls and dtls protocols and technologies around them." https://gitlab.com/gnutls/ gnutls/, 2019

[5] "Cve-2016-2182," http://cve.mitre.org/cgi-bin/cvename.cgi?name= CVE-2016-2182, 2016.

[6] "Cve-2016-2113," http://cve.mitre.org/cgi-bin/cvename.cgi?name= CVE-2016-2113, 2016.

[7] M. Georgiev, S. Iyengar, S. Jana, R. Anubhai, D. Boneh, and V. Shmatikov, "The most dangerous code in the world: validating SSL certificates in non-browser software," in CCS'12, Raleigh, NC, USA, October 16-18, 2012, 2012, pp. 38-49.

[8] J. Clark and P. C. van Oorschot, "Sok: SSL and HTTPS: revisiting past challenges and evaluating certificate trust model enhancements," in SP 2013, Berkeley, CA, USA, May 19-22, 2013, 2013, pp. 511-525.

[9] C. Brubaker, S. Jana, B. Ray, S. Khurshid, and V. Shmatikov, "Using frankencerts for automated adversarial testing of certificate validation in SSL/TLS implementations," in SP 2014, Berkeley, CA, USA, May 18-21, 2014, 2014, pp. 114-129.

[10] A. Delaitre, B. Stivalet, E. Fong, and V. Okun, "Evaluating bug finders - test and measurement of static code analyzers," in COUFLESS 2015 Florence, Italy, May 23, 2015, 2015, pp. 14-20.

[11] B. He, V. Rastogi, Y. Cao, Y. Chen, V. N. Venkatakrishnan, R. Yang, and Z. Zhang, "Vetting SSL usage in applications with SSLINT," in SP 2015, San Jose, CA, USA, May 17-21, 2015, 2015, pp. 519-534.

[12] I. Yun, C. Min, X. Si, Y. Jang, T. Kim, and M. Naik, "Apisan: Sanitizing API usages through semantic cross-checking," in USENIX Security 16, Austin, TX, USA, August 10-12, 2016., 2016, pp. 363-378.

[13] D. A. Ramos and D. R. Engler, "Under-constrained symbolic execution: Correctness checking for real code," in USENIX Security 15, Washington, D.C., USA, August 12-14, 2015., 2015, pp. 49-64.

[14] "Curl: A command line tool and library for transferring data with url syntax." https://github.com/curl/curl, 2019.

[15] “Cve-2015-0288," https://www.cvedetails.com/cve/CVE-2015-0288/, 2015.

[16] "Cve-2014-3567," http://cve.mitre.org/cgi-bin/cvename.cgi?name= CVE-2014-3567, 2015.

[17] B. Cheng and W. W. Hwu, "Modular interprocedural pointer analysis using access paths: design, implementation, and evaluation," in PLDI 2000, Vancouver, Britith Columbia, Canada, June 18-21, 2000, 2000, pp. $57-69$.

[18] A. Bessey, K. Block, B. Chelf, A. Chou, B. Fulton, S. Hallem, C. Gros, A. Kamsky, S. McPeak, and D. R. Engler, "A few billion lines of code later: using static analysis to find bugs in the real world," Commun. ACM, vol. 53, no. 2, pp. 66-75, 2010 .

[19] A. Arusoaie, S. Ciobaca, V. Craciun, D. Gavrilut, and D. Lucanu, "A comparison of open-source static analysis tools for vulnerability detection in c/c++ code," in SYNASC 2017, 2017, pp. 161-168.

[20] S. Amann, H. A. Nguyen, S. Nadi, T. N. Nguyen, and M. Mezini, "A systematic evaluation of static api-misuse detectors," IEEE Transactions on Software Engineering, pp. 1-1 (Early Access), 2018.

[21] M. Kassab, J. F. DeFranco, and P. A. Laplante, "Software testing: The state of the practice," IEEE Software, vol. 34, pp. 46-52, 2017. 